Institute of Science in Society

Science

Society

Sustainability

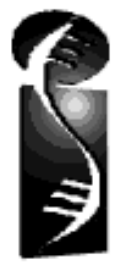

ISIS

Relevant Links:

- i-sis news \#6

- Xenotransplantation - How Bad Science and Big Business Put the World at Risk from Viral Pandemics

- The Organic Revolution in Science and Implications for Science and Spirituality

- Use and Abuse of the Precautionary Principle

- i-sis news \#5

Kybernetes 26, 265-276, 1997.

\title{
Quantum Coherence and Conscious Experience
}

\author{
Mae-Wan Ho \\ Bioelectrodynamic Laboratory, Open University \\ Walton Hall, Milton Keynes, MK7 6AA, U.K.
}

- Abstract

- How to understand the organic whole

- The organism as a vibrant sentient whole

- Quantum coherence and body consciousness

- Quantum Coherence and the Binding Problem

- Coherent information storage and qualia of perception

- Quantum coherence and the macroscopic wave function of the conscious being

- Acknowledgments

- References 


\begin{abstract}
I propose that quantum coherence is the basis of living organization and can also account for key features of conscious experience -- the "unity of intentionality", our inner identity of the singular "I", the simultaneous binding and segmentation of features in the perceptive act, the distributed, holographic nature of memory, and the distinctive quality of each experienced occasion.
\end{abstract}

\title{
How to understand the organic whole
}

Andrew's ${ }^{[1]}$ assessment that brain science is in a "primitive" state is, to some extent, shared by Walter Freeman ${ }^{[2]}$, who, in his recent book, declares brain science "in crisis". At the same time, there is a remarkable proliferation of Journals and books about consciousness, which brain science has so far failed to explain, at least in the opinion of those who have lost faith in the conventional reductionist approach. One frequent suggestion is the need for quantum theory, though the theory is interpreted and used in diverse, and at times conflcting ways by different authors.

I believe that the impasse in brain science is the same as that in all of biology: we simply do not have a conceptual framework for understanding how the organism functions as an integrated whole. Brain science has been more fortunate than many other areas in its long established multi-disciplinary practice, which is crucial for understanding the whole. In particular, the development of noninvasive/nondestructive imaging techniques has allowed access to the living state, which serves to constantly remind the reductionists among us of the ghost of the departed whole. The images obtained from the ultra sensitive, and hence truly noninvasive magnetic tomography ${ }^{[3]}$ are captivating. Analyzing such data presents an even greater challenge than the multichannel eeg data obtained by Freeman and Barrie ${ }^{[4]}$. Both kinds of data are showing up largescale spatiotemporal coherence of brain activities that cannot be satisfactorily explained by conventional mechanisms. The brain functions, not as a collection of specialized brain cells, but as a coherent whole. That is surely one good reason to seek alternative perspectives that would help us understand the organic whole.

How the brain functions as a coherent whole is inseparable from how the organism functions as a coherent whole. It is the same question, stated eloquently by Joseph Needham in Order and Life [5], and by Schrödinger in What is Life? [6], that has exercised generations of biologists and physicists dissatisfied with the mechanistic approach.

Inspired by this long line of distinguished dissidents, I began to work towards a theory of the organism based on empirical and theoretical findings across the disciplines ${ }^{[7-13]}$. The theory starts from thermodynamic considerations of energy storage in a space-time structured system under energy flow, which, by dynamic closure, creates the conditions for quantum coherence. This effectively frees the organism from thermodynamic constraints so that it is poised for rapid, specific intercommunication, enabling it to function as a coherent whole. In the ideal, the organism is a quantum superposition of coherent activities, with instantaneous (nonlocal) noiseless intercommunication throughout the system. 
I do not think quantum theory per se will lead us through the mechanistic deadlock to further understanding. Instead, we need a thoroughly organicist way of thinking that transcends both conventional thermodynamics and quantum theory ${ }^{[7,12]}$. I have focussed on the notion of quantum coherence and the attendant nonlocal intercommunication as the expression of the radical wholeness of the organism, where global and local are mutually entangled, and every part is as much in control as it is sensitive and responsive.

In this paper, I shall briefly summarize the arguments for quantum coherence in the living system, then go on to explore how certain key features of conscious experience may be understood. I suggest that the wholeness of the organism is based on a high degree of quantum coherence. Quantum coherence underlies the "unity of intentionality" [2] and our inner identity of the singular "I". It may account for binding and segmentation in the perceptive act, the distributed, holographic nature of memory, and the distinctive quality of each experienced occasion.

\section{The organism as a vibrant sentient whole}

Organisms overcome the immediate constraints of thermodynamics in their capacity to store mobilizable energy, which circulates through a cascade of cyclic processes within the system before it is dissipated [11-13]. The dynamic closure of circulating energy constitutes a life cycle. Within the life cycle, coupled cylic processes span the entire gamut of space-times from the local and fast (or slow) to the global and slow (or fast). This enables energy to be readily shared throughout the system, from local to global and vice versa, which is why we can have energy at will. But how is energy mobilization so well-coordinated? That is partly a direct consequence of the energy stored, which renders the whole systemexcitable, or highly sensitive to specific weak signals. Weak signals originating anywhere within or outside the system will propagate throughout the system and become amplified, often into macroscopic action. Intercommunication can proceed very rapidly, in particular, on account of the liquid crystalline structure of the cells and the connective tissues ${ }^{[14]}$.

Connective tissues make up the bulk of all multicellular animals. They are flexible, highly responsive, yet ordered phases which are connected, via transmembrane proteins to the intracellular matrices of individual cells $[\mathbf{1 5}, \mathbf{1 6}]$. The extracellular and intracellular matrices together constitute an excitable continuum for rapid intercommunication permeating the entire organism, enabling it to function as a coherent whole ${ }^{[13]}$. The existence of this liquid crystalline continuum has been directly demonstrated in all live organisms by a noninvasive optical imaging technique recently discovered in my laboratory [17-19]. It constitutes a "body consciousness" that precedes the nervous system in evolution [16]; and I suggest, it still works in tandem with, and independently of the nervous system (see next Section). This body consciousness is the basis of sentience, the pre-requisite for conscious experience that involves the participation of the intercommunicating whole of the energy storage domain. In

the limit of the coherence time and coherence volume of energy storage, intercommunication is instantaneous and nonlocal. Because energy is stored over all modes, the organism possesses a complete range of coherence times and coherence volumes ${ }^{[7]}$.

The life cycle, with its complex of coupled cyclic processes, forms a heterogeneous, 
multidimensional and entangled space-time which structures experience. In the ideal, it is a quantum superposition of coherent space-time modes, constituting a pure state that maximizes both local freedom and global cohesion $[7,12,13]$ in accordance with the factorizability of the quantum coherent state $[20]$. Quantum coherence gives rise to correlations between subsystems which resolves neatly into products of the self-correlations so that the sub-systems behave as though they are independent of one another. One can also picture the organism as a coherent quantum electrodynamical field of many modes, with an uncertainty relationship between energy and phase ${ }^{[21]}$,

\section{DnDf e h}

So, when phase is defined, energy is indeterminate, and vice versa. That may be of fundamental importance to the flexibility and adaptability of the living system.

In quantum optics and quantum electrodynamic theory the coherent state is asymptotically stable ${ }^{[22]}$. Hence, the pure coherent state is an ideal attractor or end state towards which the system tends to return on being perturbed ${ }^{[23]}$. There is abundant evidence of macroscopic activities with collective phases in the spectrum of biological rhythms, many of which tend towards integral phase relationships with one another $[\mathbf{7}, 24]$. There are also examples of collective activities that may involve phase correlations over entire populations ${ }^{[25,26]}$. As the coherence times of living processes span more than 20 orders of magnitude from 10-14s for resonant energy transfer to $107 \mathrm{~s}$ for circannual cycles, a pure coherent state for the entire system would be a many-mode quantum electrodynamical field with a collective phase over all modes. It may be attainable only under very exceptional circumstances, as during an aesthetic or religious experience when the "pure duration" (see later) of the here and now becomes completely delocalized in the realm of no-time and no-space [7]. Nevertheless, quantum coherence can exist to different degrees or orders [20]; and

I suggest that the wholeness of the organism is based on a high degree of quantum coherence. It constitutes Freeman's "unity of intentionality" [2] -- the pre-requisite to conscious experience.

\section{Quantum coherence and body consciousness}

From the perspective of the whole organism, the brain's primary function may be the mediation of coherent coupling of all subsystems, so the more highly differentiated or complex the system, the bigger the brain required. Substantial parts of the brain are indeed involved in integrating inputs from all over the body, and over long time scales. But not all the processing that goes on in the brain is involved in the coherent coordination of subsystems, for this coordination seems instantaneous by all accounts.

Thus, during an olfactory experience, slow oscillations in the olfactory bulb are in phase with the movement of the lungs ${ }^{[4]}$. Similarly, the coordinated movement of the four limbs in locomotion is accompanied by patterns of activity in the motor centers of the brain which are in phase with those of the limbs ${ }^{[27]}$. That is a remarkable achievement which physiologists and neuroscientists alike have taken too much for granted. The reason macroscopic organs 
such as the four limbs can be coordinated is that each is individually a coherent whole, so that a definite phase relationship can be maintained among them. The hand-eye coordination required for the accomplished pianist is extremely impressive, but depends on the same inherent coherence of the subsystems which, I suggest, enables instantaneous intercommunication to occur. There simply isn't time enough, from one musical phrase to the next, for inputs to be sent to the brain, there to be integrated, and coordinated outputs to be sent back to the hands (c.f. Hebb ${ }^{[28]}$ ).

I raised the posssibility, above, that a "body consciousness" works in tandem with, but independently of the "brain consciousness" constituting the nervous system. I suggest that instantaneous coordination of body functions is mediated, not by the nervous system, but by the body consciousness inhering in the liquid crystalline continuum of the body. Ho and Knight ${ }^{[29]}$ following Oschman ${ }^{[16]}$, review evidence suggesting that this liquid crystalline continuum is responsible for the direct current (DC) electrodynamical field, permeating the entire body of all animals, that Becker ${ }^{[30]}$ and others have detected. Becker has further demonstrated that the DC field has a mode of semi-conduction that is much faster than nervous conduction. During a perceptive event, local changes in the DC field can be measured half a second before sensory signals arrive in the brain, suggesting that the activities in the brain may be pre-conditioned by the local DC field.

Up to $70 \%$ of the proteins in the connective tissues consist of collagens that exhibit constant patterns of alignment, as characteristic of liquid crystals. Collagens have distinctive mechanical and dielectric properties that render them very sensitive to mechanical pressures, changes in $\mathrm{pH}$, inorganic ions and electromagnetic fields $\left.{ }^{[29}\right]$. In particular, a cylinder of bound water surrounds the triple-helical molecule, giving rise to an ordered array of bound water on the surface of the collagen network that supports rapid "jump conduction" of protons. Proteins in liquid crystals have coherent residual motions, and will readily transmit weak signals by proton conduction, or as coherent waves [31]. Thus, extremely weak electromagnetic signals or mechanical disturbances will be sufficient to set off a flow of protons that will propagate throughout the body, making it ideal for intercommunication in the manner of a proton-neural network ${ }^{[32]}$.

The liquid crystalline nature of the continuum also enables it to function as a distributed memory store. The proportion of bound versus free water on the surfaces of proteins are known to be altered by conformation changes of the proteins. Proteins undergo a hierarchy of conformational changes on a range of time scales as well as different energies. Conformers are clustered in groups that are nearly isoenergetic, with very low energetic barriers between them ${ }^{[33]}$. They can thus be triggered to undergo global conformational changes that will, in turn, alter the structure of bound water. As the bound water forms a global network in association with the collagen, it will have a certain degree of stability, or resistance to change. The corollary is that it will retain tissue memory of previous experiences. The memory may consist partly of dynamic circuits, the sum total of which constituting the DC body field. Thus, consciousness is distributed throughout the entire body, brain consciousness being embedded in body consciousness. Brain and body consciousness mutually inform and condition each other. The unity of intentionality is a complete coherence of brain and body. 


\section{Quantum Coherence and the Binding Problem}

So it is that we perceive ourselves as a singular "I" intuitively, despite the extremely diverse multiplicity of tissues, cells and molecules consituting our being (c.f. Schrödinger [6]). Quantum coherence entails a plurality that is singular, a multiplicity that is a unity. The "self" is the domain of coherence [7], a pure state or pure duration that permeates the whole of our being, much as Bergson ${ }^{[34]}$ has described.

It is because we perceive ourselves as a singular whole that we perceive the real world as colour, sound, texture and smell, as a unity all at once. Sounds presented in linear sequences are recognized as speech or music, much as objects in motion are recognized as such, rather than as disconnected configurations of light and shadow. How is this unity structured so that not only can we recognize whole objects, but distinguish different objects in our perceptual field? That is the problem of binding and reciprocally, of segmentation ${ }^{[35}$.

Detailed investigations over the past decades have revealed that there are many cells which respond to isolated features such as edges or bars in the visual cortex, but no special cells have been found to respond to higher categories ${ }^{[1]}$, such as squares or cubes for example. There is simply no "grandmother cell" that integrates the separate features. So how are the separate features bound into a whole? And how is it that we can bind features correctly so that they belong to the same object in the real world? For example, how do we see correctly, a red rose in a yellow vase and not a yellow rose in a red vase? It turns out that timing is of the essence.

Freeman ${ }^{[2]}$ and his coworkers carried out simultaneous recordings with an array of 64 electrodes covering a large area of the rabbit cortex, and found oscillations that are coherent over the entire array. These tend to vary continually or abruptly, but when they change, they do so in the same way over the whole area. The amplitudes will differ, but the pattern of discharges is simultaneous and uniform. He concludes, "This spatial coherence indicates that the oscillation is a macroscopic property of the whole area, that all the neurons in the neuropil share it, and that the same frequency holds at each instant everywhere."(p. 57). Gray et al ${ }^{[36]}$ recorded simultaneously from pairs of neuronal units whose outputs might be subject to binding. These are in the same or different cerebral hemispheres and responded to the same or different sensory modalities. They found that throughout the wide range of situations, the characteristic feature of paired discharges that are suitable for subsequent binding is a high degree of coincidence in time. It seems that the nervous system produces "simultaneity as an aid to subsequent binding."

Singer [37] has also found evidence of simultaneous oscillations in separate areas of the cortex, accurately synchronized in phase as well as frequency. He suggests that the oscillations are synchronized from some common source, but Freeman's group, using a large array of electrodes (see above) failed to identify any obvious source. As Andrew ${ }^{[1]}$ points out, the accuracy of phase agreement is far too perfect for the synchronizing to spread by

normal neural transmission, and he favours some kind of optical signal transmitted by water trapped in microtubules acting as optical fibres ${ }^{[38,39]}$.

If, however, the system is coherent to begin with, then a genuine nonlocal simultaneity may 
be involved. The present precision of recording is insufficient to distinguish between instantaneous simultaneity and propagation at the speed of light. As is well-known, there is no time-like separation within the coherence volume, and no space-like separation within the coherence time, so apparent "communication" is instantaneous, and synchrony can be established with no actual delay. This simultaneity may be mediated and gated by the DC body field mentioned above. That can easily be tested by repeating the measurements carried out by Becker ${ }^{[30]}$.

The clue to both binding and segmentation is in the accuracy of phase agreement of the spatially separated brain activities. That implies the nervous system (or the body field) can accurately detect phase, and is also able to control phase coherence. I have already alluded to the importance of phase information in coordinating limb movements during locomotion and other aspects of physiological functioning, so it is not surprising that the nervous system should be able to accurately detect phase. The degree of precision may be estimated by considering our ability to locate the source of a sound by stereophony. Some experimental findings show that the arrival times of sound pulses at the two ears can be discriminated with an accuracy of a very few microseconds ${ }^{[1]}$. For detecting a note in middle $\mathrm{C}$, the phase difference in a microsecond is $4.4 \times 10-4$. Accurate phase detection is characteristic of a system operating under quantum coherence. Could it be that phase detection is indeed a key feature of conscious experience?

Marcer [ 40, 41] has proposed a "quantum holographic" model of consciousness in which perception involves the conversion of an interference pattern (between a coherent wave-field generated by the perceiver and the wave-field reflected off the perceived) to an object image that is coincident with the object itself. This is accomplished by a process known as phase conjugation, whereby the wave reflected from the object is returned (by the perceiver) along its path to form an image where the object is situated. The perceiving being is into the act of perceiving, as Freeman [2] observes. Endogenously generated coherent waves or activities, therefore, function as precise gating, on the basis of phase information, to bind and segment features as appropriate. In the act of perceiving, the organism also perceives itself situated in the environment, through active phase conjugation. As Gibson ${ }^{[42]}$ remarks, perception and proprioception are one and the same. Within the perceptive realm of the organism, there will always be an image of the self as the focus of "prehensive unification" [43], to which all features in the environment are related. Marcer's quantum holographic model of self-consciousness would involve an image of the self coincident with the organism itself, so "self" and "other" are simultaneously defined. What is the source of the coherent wave-field generated by the perceiver? Could it be the body field itself? Or the body field as modulated by the nervous system? Again, this could be subject to empirical investigation.

One thing seems clear. Quantum coherent systems can bind and segment simultaneously and nonlocally by virtue of their factorizability (see above), which is how living processes are organized. Circulation, metabolism, muscular and nervous acitivities all go on simultaneously and independently, yet nevertheless cohering into a whole. A multitude of bound and segmented simultaneities are created in the act of experiencing, which define the here and now. These simultaneities are nonlocal and heterogeneous. They contain further simultaneities within and become entangled as they cascade through a quasi-continuum of space-times. The here and now is, therefore, not a flat instantaneity, nor a travelling razor blade dividing past from future (c.f. Gibson ${ }^{\left[\mathbf{4 2}^{2}\right.}$ ). Instead, it is the grain of experiencing -- a 
labyrinth of commuting and non-commuting simultaneities within simultaneities out of which hesitations we weave our futures.

\section{Coherent information storage and qualia of perception}

The conscious being initiates and gates experience and determines the content of the experience, so it is that two people can experience the same music simultaneously, one with the highest rapture, and the other, the utmost indifference. According to Gibson [42], objects in the environment provide "affordances" which are selected by the subject in the act of perceiving. The information goes into "resonant circuits in the brain" from which "effectivities" flow, ultimately as "object-oriented actions" complementary to the affordances. Thus, the quality of each perception is coloured by all that has gone before ${ }^{[2]}$, the brain does more than coordinate subsystems of the body, it forms images (or, at any rate, takes part in forming images), and stores them for future reference.

The stored information, or memory system, is generally found to be distributed over the entire brain, perhaps in the form of "reverberations" or circuits that "mediate" responses to stimuli and initiate actions. Thus, in contrast to the rapidity with which simultaneity can be established in different parts of the brain, half a second is required for the subject's brain to become "aware" (as evidenced by its electrical activities) that something has happened, although the subject automatically back-dates it to make up for the delay (see earlier). Freeman's view is that the delay is the time needed for "propagation of a global state transition through a forebrain to update the state of the intentional structure by learning." $(\mathrm{p}$. 83). In other words, that is the time taken to reorganize the whole system.

There does appear to be a circulating activity in a network consisting of different brain structures, and transmitted between various regions in a highly organized fashion. These circulating activities, modified by sensory inputs, are thought to be responsible for 'short-term' memory, which becomes long-term memory by causing structural chemical changes ${ }^{[44]}$. However, it would be a mistake to suppose that memory is thereby 'fixed' once and for all. Molecules in the brain, as in all of the rest of the body, are subject to metabolic turnover. So, it is more realistic to suppose that so-called 'long-term' memory is subject to the same dynamic modification and reconstitution as short-term memory, and that short-term and long term are simply the ends of a continuum that extends from the most microscopic "here and now" to the individual's entire life-span and beyond. It is this dynamic information store, distributed over a whole gamut of timescales that underlies the distinctive quality of each experience, for the experiencing being is constantly being renewed and updated.

Thus, Freeman ${ }^{[2]}$ and his coworkers found that rabbits trained to distinguish odours have patterns of brain activities for each odour that are never twice the same in any one session for any animal. And each animal has its own repertoire of patterns which evolve in successive trials. Far from being disconsolate, the experiments have given Freeman new insights into the unity of intentionality in that every perception is influenced by all that has gone before. Constant stimulus-response relationships are not mediated by correspondingly constant cause and effect associations of brain activities. In contrast to the microscopic patterns carried by a few sensory neurons which differ consistently with each smell, the macroscopic spatial 
patterns in the olfactory bulb are distributed over the entire bulb for every odour, and "did not relate to the stimulus directly but instead to the meaning of the stimulus."(p. 59) So, when reward was switched between two odours, the patterns of activities changed for both odours, as also did the control patterns without odour in background air. The patterns changed whenever a new odour was added to the repertoire. There is no mosaic of compartments in the olfactory memory in the bulb. It is a seamless information store.

All the evidence points to a dynamic maintenance and recreation of memory over all time scales. There is a transfer of information to ever longer and longer time scales exactly in the way that energy gets transferred in cascades of processes of increasingly larger space-times ${ }^{[7]}$. In the transfer of memory, different memories also become entangled in the reconstituion of the whole, thus continually redefining a unique here and now. One never ceases to write and overwrite one's biography -- it is a tissue of reconstructions. There is no sharp distinction between the here and now and what has gone before. 'Past' simultaneities over-arch the 'now' and extend beyond while further simultaneities are seeded within the 'now'.

Strong evidence that memory storage is delocalized, at least over the whole brain, is the finding that it is able to survive large brain lesions. This has already led a number of people to suggest that memory storage is holographic, in the same way that perception is holographic, so that the whole can be reconstructed from even a small part, albeit with less detail. As Langfield ${ }^{[45]}$ points out, holography enables complex information to be retrieved simply by generating a regular wave without any informational content. Of course, the same regular or coherent wave is instrumental in creating and coding the complex information in the first place. Likely candidates for coherent reference waves are considered to include alpha waves and waves generated by the hippocampus. Langfield has proposed a model in which memory is encoded by coherent waves from the hippocampus interacting with sensory inputs and undergoing a phase change. These modulated "object" waves are then recombined with the reference waves to form an interference pattern in the pyrimidal cells of the hippocampus, from which a "reconstructed wavefront" is projected to other parts of the brain to generate the circulating patterns of activity that constitute "short-term memory". This short-term memory is thought to be consolidated during sleep, whereas the alpha rhythms occurring during states of relaxation are believed to play a special role in memory retrieval.

Holographic memory storage is orders of magnitude more efficient than any model that makes use of "representations" because holographic memory employs actual physical simulations of processes ${ }^{[\mathbf{4 0}, \mathbf{4 1}]}$ and do not require lengthy sequences of arbitrary coding and decoding of isolated bits. Marcer suggests that the brain stores experienced holographic spatio-temporal patterns and compares stored with new patterns directly, recognition and learning being reinforced in "adaptive resonance", thus also making for much faster processing. As mentioned before, the liquid crystalline continuum supporting the body field may also take part in memory storage, although this possibility has never been seriously considered. Laszlo ${ }^{[47]}$ goes even further to suggest that much of memory may be stored in an ambient, collective holographic memory field delocalized from the individual; and that memories are only accessed by the brain from the ambient field. 


\section{Quantum coherence and the macroscopic wave function of the conscious being}

If quantum coherence is characteristic of the organism as conscious being, as I have argued here, then the conscious being will possesss something like a macroscopic wave-function. This wave function is ever evolving, entangling its environment, transforming and creating itself anew [7]. I agree with Bohm and Hiley's [46] ontological interpretation of quantum theory to the extent that there is no collapse of the wave function. In their model, the wave function, with quantum potential playing the role of active information to guide the trajectories of particles, simply changes after interaction to become a new one. The possibility remains that there is no resolution of the wave functions of the quantum objects after interacting. So one may remain entangled and indeed, delocalized over past experiences (i.e., in Lazlo's ambient field ${ }^{[46]}$ ). Some interactions may have time scales that are extremely long, so that the wave function of interacting parties may take a correspondingly long time to become resolved, and largescale nonlocal connectivity may be maintained.

What would our wave function look like? Perhaps it is an intricate supramolecular orbital of multidimensional standing waves of complex quantum amplitudes. It would be rather like a beautiful, exotic flower, flickering in and out of many dimensions simultaneously. That would constitute our quantum holographic self, created from the entanglements of past experiences, the memory of all we have suffered and celebrated, the totality of our anxieties and fears, our hopes and dreams.

\section{Acknowledgments}

I thank Peter Marcer, David Knight, Walter Freeman and Brian Goodwin for stimulating discussions. 


\section{References}

1. Andrew, A. M. (1995). "The decade of the brain -- some comments", Kybernetes 24, 54-57.

2. Freeman, W.J. (1995). Societies of Brains. A Study in the Neuroscience of Love and Hate, Lawrence Erlbaum Associates, Hove.

3. Iaonnides, A. A. (1994). "Estimates of brain activity using magnetic field tomography and large scale communication within the brain", in Bioelectrodynamics and Biocommunication (M.W. Ho, F.A. Popp and U. Warnke, eds.), World Scientific, Singapore.

4. Freeman, W.J. and Barrie, J.M. (1994). "Chaotic oscillations and the genesis of meaning in cerebral cortex". In Temporal Coding in the Brain (G. Bizsaki, ed.), Springer-Verlag, Berlin.

5. Needham, J. (1936). Order and Life, MIT Press, Cambridge, Mass.

6. Schrödinger, E. (1944). What is Life? Cambridge University Press, Cambridge.

7. M.W. Ho. (1993). The Rainbow and the Worm: The Physics of Organisms, World Scientific, Singapore.

8. Ho, M.W. (1994a). "What is (Schrödinger's) negentropy?", Modern Trends in BioThermoKinetics 3, 50-61.

9. See Ho, M.W. ed. (1995a) Bioenergetics, S327 Living Processes, An Open University Third Level Science Course, Open University Press, Milton Keynes.

10. Ho, M.W. (1995b). "Bioenergetics and the coherence of organisms",Neural Network World 5, 733-750.

11. Ho, M.W. (1996b). "Bioenergetics and biocommunication", in IPCAT95 Proceedings (R. Paton, ed.), World Scientific, Singapore, in press.

12. Ho, M.W. (1996a). "The biology of free will", J. Consciousness Studies 3, 231-244.

13. Ho, M.W. (1996c). "Towards a theory of the organism" (submitted).

14. Ho, M.W., Haffegee, J., Newton, R., Zhou, Y.M., Bolton, J. and Ross, S. (1996). "Organisms as polyphasic liquid crystals", Bioelectrochemistry and Bioenergetics (in press).

15. Oschman, J. L.(1984). "Structure and properties of ground substances", Am. Zool. 24, 199-215.

16. Oschman, J.L. (1993). "A Biophysical basis for acupuncture", private manuscript.

17. Ho, M.W. and Lawrence, M. (1993). Interference colour vital imaging -- a novel noninvasive technique. Microscopy and Analysis, September, 26.

18. Ho, M.W. and Saunders, P.T. (1994). Liquid crystalline mesophases in living organisms. In Bioelectromagnetism and Biocommunication (M.W. Ho, F.A. Popp and U. Warnke, eds.). World Scientific, Singapore.

19. Newton, R., Haffegee, J. and Ho, M.W. (1995). "Colour-contrast in polarized light microscopy of weakly birefringent biological specimens", J. Microscopy (in 4.

20. Glauber, R.J. (1969). "Coherence and quantum detection", in Quantum Optics (R.J. Glauber, ed.), Academic Press, New York.

21. Preparata, G. (1994). "What is quantum physics? bak to the QFT of Planck, Einstein and Nernst", Lecture given at IX Winter School on Hadron Physics, Folgaria (Italy). 
22. Goldin, E. (1982). Waves and Photons, An Introduction to Quantum Optics, John Wiley \& Sons, New York.

23. Ho, M.W. (1996c). "Bioenergetics, Biocommunication and Organic Space-time", inProceedings of the British Computing Society Conference on Living Computer, Greenwich, London.

24. Breithaupt, H. (1989). "Biological rhythms and communications", in Electromagnetic Bioinformation 2nd ed. (F.a. Popp, U. Warnke, H.L. Konig and W. Peschka, eds.), pp. 18-41, Urban \& Schwarzenberg, Berlin.

25. Strogatz, S.H. and Mirollo, R.E. (1988). "Collective synchronisation in lattices of non-linear oscillators with randomness", J. Phys. A: Math. Gen. 21, L699-L705.

26. Ho, M.W. Xu, X., Ross, S. and Saunders, P.T. (1992b). In Advances in Biophotons Research (F.A. Popp, K.H. Li and Q. Gu, eds.), pp. 287-306, World Scientific, Singapore.

27. Kelso, J.A.S. (1991). "Behavioral and neural pattern generation: The concept of neurobehavioral dynamical systems", in Cardiorespiratory and Motor Coordination (H.P. Koepchen and T. Huopaniemi, eds.), pp. 224-234, Springer-Verlag, Berlin.

28. Hebb, D.O. (1958). A Textbook of Psychology, W.B. Saunders, Philadelphia.

29. Ho, M.W. and Knight, D. (1996). Collagen liquid crystalline phase alignment and the DC body field of consciousness (in preparation).

30. Becker, R.O. (1990). Cross Currents. The Promise of Electromedicine, The Perils of Electropollution, Jeremy P. Tarcher, inc. Los Angeles.

31. Mikhailov, A.S. and Ertl, G. (1996). "Nonequilibrium structures in condensed systems" Science 272, 1596-1597

32. Welch, G.R. and Berry, M.N. (1985). "Long-range energy continua and the coordiantion of multienzyme sequences in vivo", in Organized Multienzyme Systems (G.R. Welch, ed.), Academic Press, New York.

33. Welch, G.R. ed. (1986). Fluctuating Enzyme, John Wiley \& Sons, New York.

34. Bergson, H. (1916). Time and Free Will. An Essay on the Immediate Data of Consciousness (F.L. Pogson, trans.), George Allen \& Unwin, Ltd., New York.

35. Hardcastle, V. G. (1994). "Psychology's 'binding problem' and possible neurobiological solutions. $J$. Consciousness Studies 1, 66-90.

36. Gray, C.M., Konig, P., Engel, A.K. and singer, W. "Oscillatory responses in cat visual cortex exhibit inter-columnar synchronization which reflects global stimulus properties", Nature 33, 334-337.

37. Singer, W. (1990). "Self-organization of cognitive structures", in The Principles of Design and Operation of the Brain, (J. Eccles and O. Creutzfeld, eds.), Springer, Berlin.

38. Hameroff, S. and Penrose, R. (1995). "Orchestrated reduction of quantum coherence in brain microtubules, a model of consciousness", Neural Network World 5, 793-812.

39. Jibu, M., Hagan, S., Hameroff, S.R., Pribram, K.H., Yasue, K. (1994). "Quantum optical coherenc in cytoskeletal microtubules: implications for brain function", Biosystems 32, 95-209.

40. Marcer, P.J. (1992). "Designing new intelligent machines -- the Huygens' machine. CC-AI Journal 9 , 373-394. 
41. Marcer, P.J. (1995). "The need to define consciousness -- a quantum mechanical model", Symposium, (P.J. Marcer and A.M. Fedorec, eds.), University of Greenwich, pp. 23-15.

42. Gibson, J.J. (1966). The Ecological Approach to Visual Perception, MIT ress, Mass.

43. Whitehead, A.N. (1925). Science and the Modern World, Penguin Books, Harmondsworth.

44. Berzeano, M. (1977). "The activity of neuronal network in memory consolidation", in Neurobiology of Sleep and Memory (R.R. Drucker-Colin and J.L. McGaugh, edsl), Acadeic Press, New York.

45. Langfield, P.W. (1976). "Synchronous EEG rhythms: Their nature and their possible functions in memory, information transmission and behaviour" in Molecular and Functional Neurobiology (W.H. Gispen, ed.), Elsevier, Amsterdam.

46. Bohm, D. and Hiley, B.J. (1993). The Undivided Universe, Routledge, London.

47. Laszlo, E. (1995). The Interconnected Universe, World Scientific, Singapore.

The Institute of Science in Society

PO Box 32097, London NW1 OXR

Tel: 44 -020-7380 0908

Material on this site may be reproduced in any form without permission, on condition that it is accredited accordingly and contains a link to http://www.i-sis.org.uk/

mirrored in California inside:

http://www.ratical.org/co-globalize/MaeWanHo/ 